\title{
How to Share and Reuse Learning Resources: The ARIADNE Experience
}

\author{
Joris Klerkx, Bram Vandeputte, Gonzalo Parra, José Luis Santos, \\ Frans Van Assche, and Erik Duval \\ Katholieke Universiteit Leuven \\ Departement computerwetenschappen \\ Celestijnenlaan 200A, 3000 Leuven, Belgium \\ http://hmdb.cs.kuleuven.be
}

\begin{abstract}
ARIADNE is a European foundation that aims to foster "Share and Reuse" of learning resources. To support this goal, ARIADNE has created an infrastructure for managing learning objects in an open and scalable way. This paper describes the technical approach behind our open, standards based infrastructure, how content providers can connect to it, and the value they can derive from doing so. As such, the abundance that we help to unlock will act as a platform for innovation by tool developers, trainers and teachers and learners themselves.
\end{abstract}

Keywords: ariadne, learning objects, metadata, reuse, interoperability.

\section{Introduction}

ARIADNE is a European foundation that aims to foster "Share and Reuse" of learning resources. Reusing digital resources for learning has been a goal for several decades, driven by potential time savings and quality enhancements. To support this goal, ARIADNE has created a standards-based infrastructure for managing learning objects in an open and scalable way 33. The overall goal of our infrastructure is to provide flexible, effective and efficient access to large-scale collections in a way that goes beyond what typical search engines provide 11 . Therefore, it provides

1. a set of services which allows the integration and management of learning objects, described in various metadata schemas in numerous repositories across the globe,

2. a toolset which allows end users to access the learning material in various ways through information visualization [16], mobile information devices [26], multi-touch displays and mash-up applications.

This paper explains how a content provider can unlock its learning objects through the ARIADNE services, thus ensuring that the material can be found in the "Global Learning Objects Brokered Exchange (GLOBE)" consortium; the largest federation worldwide which brings together content from over 70 learning object repositories provided by more than 50 organizations [13. In this way, 
this paper describes the technical approach behind our open, standards based infrastructure, how content providers can connect to it, and the value they can derive from doing so.

This papers starts of in section 2 with an introduction of the key technologies used in ARIADNE. Secondly, section 3 provides an overview of the complete ARIADNE infrastructure with all the services and tools. Section 4 shows how a content provider can make his learning material available in GLOBE through ARIADNE. An evaluation of our infrastructure is presented in section 5. Related work is presented in section [6 and we conclude this paper in section 7 .

\section{Key Technologies}

Over the last decade, ARIADNE has spent considerable effort on the development of standards and specifications for learning object repositories, with significant results. We consider the following standards and specifications as key technologies within the ARIADNE infrastructure.

\subsection{Metadata}

In order to enable flexible access to content, we need rich descriptions: these are typically called metadata. We focus on the automatic generation [21] and extraction of metadata as well as on so-called attention metadata [24] that describe interactions with content, rather than just the content itself [18]. For describing learning content, we mainly rely on IEEE LTSC LOM 14 but we support other standards like Dublin Core (DC) and ISO/IEC MLR as well, by automatically transforming metadata from one format into another (see section 3.1).

\subsection{Harvesting}

Frameworks for metadata harvesting (like OAI-PMH) enable harvesters to copy metadata from a repository and save this copy of the metadata locally 31. On top of this local copy, search services can be added to enable search in the metadata of the contents of the content providers. We typically do not copy content itself. Therefore, we do not have to deal with intellectual property rights and digital access management for the content. We do make available metadata about the content by harvesting metadata with the OAI-PMH protocol. ARIADNE asks providers to release their metadata under a CC license (CC-BY-SA-NC) to improve discoverability of learning content because all harvested metadata is exposed again in an OAI-PMH target where it can be harvested by other federations and repositories.

\subsection{Publishing}

For publishing learning objects or their metadata into the ARIADNE infrastructure, we rely on the Simple Publishing Interface (SPI) specification. This 
model [36] has been designed such that it is interoperable with (i) the "Simple Web-service Offering Repository Deposit (SWORD)" profile [2], (ii) the "Package Exchange Notification Services (PENS)" 1 and (iii) the publishing specification that was developed in the ProLearn Network of Excellence 32. With SPI, one can transfer digital educational resources from a variety of tools to the ARIADNE infrastructure.

\subsection{Querying}

The ARIADNE infrastructure provides a Simple Query Interface (SQI) [30] on top of the repository which can be used to issue queries in various query languages such as PLQL. SQI provides interoperability between search applications and various learning object repositories and is designed to support many types of search technologies. Other specifications such as SRU/SRW, O.K.I OSIDs, etc. do exist. SQI serves as a gateway to them.

\section{The ARIADNE Infrastructure}

Figure 1 shows a representation of the ARIADNE infrastructure which has three layers:

- The Storage Layer allows for storing both content and metadata in several databases.

- The Middle Layer offers persistent management of LOs and metadata through a set of services, such as an identifier service for providing unique identifiers for LOs.

- The Toolset layer hides protocols and standards for end users in a toolset that e.g. can be used for accessing LOs in the ARIADNE infrastructure.

The following sections focus on those services and tools that can be used by content providers to unlock their learning resources.

\subsection{Middle Layer}

From experience, we have learned that a set of services with specific goals are necessary for enabling share and reuse. ARIADNE has been improving these services constantly. However, with the inclusion of a number of new services such as the registry, the transformation and the identification service, ARIADNE has now reached a level of maturity leading to a much greater uptake.

The Repository services allow for the management of learning objects in an open and scalable architecture. To enable stable querying, publishing, and harvesting of digital learning material, all key technologies that have been discussed above are used in ARIADNEs repository services, which are described in detail in 33 . 

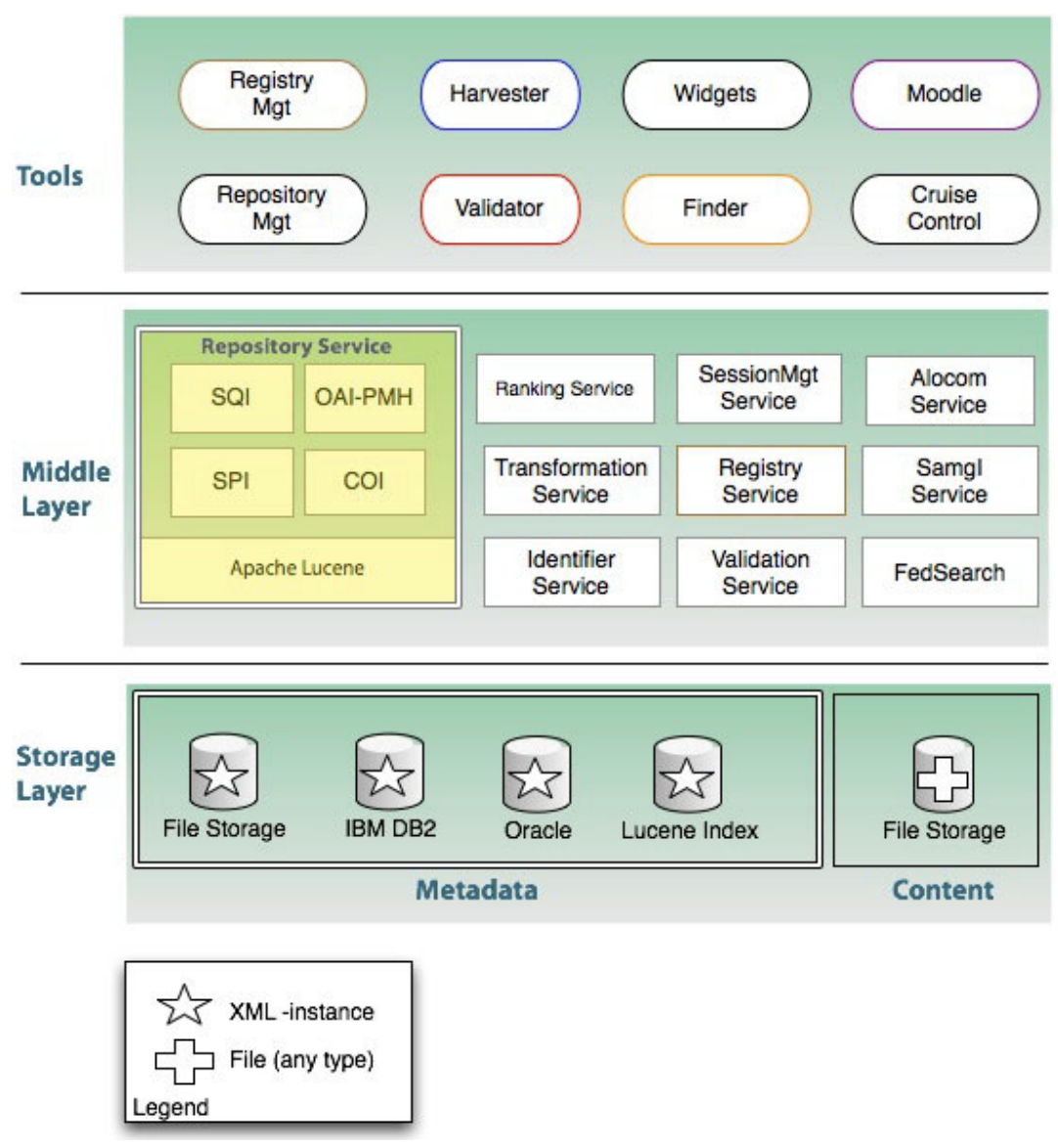

Fig. 1. The ARIADNE Infrastructure has three layers including a storage, middle and toolset layer

The Registry service is a catalog service that provides up-to-date information on learning object repositories (LORs). It provides the information necessary for systems to be able to select the appropriate protocols such as OAI-PMH, SQI, SPI, SRU/SRW [19] supported by a given learning object repository. The information is captured using the IMS LODE specification [12, that is based on the IMS Dublin Core Collections Application Profile, complemented with ISO 2146 and IEEE LOM.

The registry service facilitates interoperability between numerous learning object repositories. Besides that, creating a network of interoperable LOR registries, allows for the automatic discovery of new repositories with interesting material. Figure 2 illustrates the network of registries that has been set up in GLOBE. All information in the ARIADNE registry is exchanged with all registries in the network, with the consequence that if content provider $\mathrm{X}$ adds his target to the ARIADNE registry, client tools of the other registries like the 
EUN [5] and LACLO [6] ones, can also discover the repository. The same goes for provider $\mathrm{Y}$ who adds his target to the LACLO registry. Therefore, it can be found by client tools of the ARIADNE and the EUN registries. Such client tools could for example be harvesters that would automatically harvest all targets from a registry.

The ARIADNE harvester uses the OAI-PMH framework 31] for harvesting metadata instances from an OAI-PMH target and publishes them with the Simple Publishing Interface (SPI) into (i) a specified component of the ARIADNE storage layer, (ii) any other repository that has an SPI target on top of its repository. As an illustration, in the ASPECT eContentPlus project, the ARIADNE harvester is used to harvest metadata from thirteen ASPECT content providers. The harvester then publishes their metadata in the Learning Resource Exchange (LRE) 10. As a result, teachers can then discover these resources via the ASPECT portal. This is the client tool they are familiar with for searching learning material for their courses.

Once configured, the harvester can autonomously manage the addition, deletion and updates of metadata in a periodic and incremental way.

The validation service is available for providing validation of metadata instances against predefined application profiles, for example based on IEEE LOM. To ensure that only compliant metadata are stored in the ARIADNE repository, we use the validation service to check both the syntactic and semantic validity of the instances against the used profiles. The validation service has a modular approach, and combines different sorts of validation techniques including:

- XSD schema, mainly used for structural validation of xml metadata.

- Schematron rules 17, which are very flexible and are used for:

- verifying the presence of mandatory elements.

- checking the presence of empty attribute fields. For example, in the langstring datatype of LOM, the language of a string should be recorded in a non-empty attribute "language".

- enforcing inter-field dependencies, like conditional fields.

- checking for the correct use of the allowed terms in a vocabulary.

- validation of vcards present in the metadata with a separate vcard parser or validator.

The validation service is available as an online service where one single metadata record can be validated against the appropriate scheme. It is also integrated in the ARIADNE harvester for validating large sets of records. Reports are automatically generated which give an overview of all validation errors.

The transformation service converts metadata in one format, e.g Dublin Core (DC), into another format; e.g. the ARIADNE application profile in LOM. We need this transformation service due to the multiplicity of different metadata schemes that are used in various networks of learning object repositories [18. 
For example if we want our end users to be able to discover content, described with DC metadata, in our IEEE LOM driven query tool, we need to transform the DC to IEEE LOM first and store this representation in the ARIADNE repository.

As there are a wide variety of standards and custom formats used, the transformation service works with a growing set of transformers. These transformers transform (i) metadata from one standard to another (like DC to LOM), (ii) from one AP to another within the same standard or (iii) they can combine both. Thus for every set of metadata the appropriate transformer needs to be selected, adapted or written if it does not exist yet.

The Identification service is used to provide persistent digital identifiers to resources in the ARIADNE infrastructure. The HANDLE system 27] is used as the backend service to create globally unique, persistent and independent identifiers. This system allows the assignment, management and resolution of persistent identifiers in a distributed environment. The lower level API provided by the HANDLE system is connected to an independent service interface that provides the basic functionality for persistent storage: Create, Resolve, Update and Delete (CRUD) identifiers. The identifiers created by the service are compliant with the Universally Unique Identifier standard 15. For this purpose the Java Uuid Generator (JUG) [28] is used.

The SamgI service is able to semi-automatically generate metadata instances. Through automatic metadata generation, by extracting relevant information from contents and contexts, it is possible to significantly remove the need to fill in electronic forms when describing learning resources with metadata. This is essential if we want to make our approach scale up and become mainstream. The architecture of the SamgI service has been published in [21].

The Federated Search Service relies on SQI to offer transparent search to both ARIADNE and GLOBE. The architecture of the federated search service has been described in previous work such as [33, 34] and 30.

The Ranking service ranks search results according to Contextualized Attention Metadata (CAM) 25] which captures social data about the learning objects such as the number of times an object has been downloaded by an end user.

The ALOCOM service supports two processes: the disaggregation of learning objects into their components (text fragments, images, definitions, diagrams, tables, examples, audio and video sequences) as well as the automatic assembly of these components in real-world authoring tools [35].

\subsection{Toolset}

One of the main design principles in ARIADNE is to make everything disappear but the benefits. Within ARIADNE, we have therefore created a number of tools that are meant to make it easy to share and reuse learning resources. 
The Moodle bridge is a module for the Moodle learning management system. If this module is deployed, users of the LMS can (i) issue a query to both ARIADNE and GLOBE, (ii) import a found resource directly from the GLOBE network into the LMS, and therefore (iii) make this resource available for other users.

The ARIADNE finder is a standalone search tool that lets users search learning material and browse the results. The finder hides protocols and standards that are used in the middle layer. Among its capabilities, it supports facetted searching in ARIADNE and GLOBE. The finder can be coupled on any repository that supports the SQI standard and describes its content with IEEE LOM.

The ALOCOM Powerpoint plugin can be used while creating a presentation from within powerpoint to find interesting material in ARIADNE and GLOBE, that can be included in the presentation. Besides that, it can be used to store a created presentation into the ARIADNE repository where it is available for users from GLOBE.

Both the Moodle bridge and the ALOCOM powerpoint plugin enable users to search for relevant content from within their usual environment, which

- offers opportunities to use their context for enriching their search criteria,

- enables us to integrate the services in their familiar workflow, so that they do not need to go to a web site to call the services.

\section{From Content Provider to GLOBE}

The different components in the ARIADNE infrastructure have been explained in the previous section. Figure 2 illustrates how those services and tools can be combined by content providers $\mathrm{X}$ and $\mathrm{Y}$ to interconnect their repository with GLOBE. Imagine that all contents from provider $\mathrm{X}$ have been described by Dublin Core (DC) - Educational metadata. The provider X has already implemented an OAI-PMH [31] target on top of the repository. To connect to GLOBE, the content provider registers his OAI-PMH target into the ARIADNE Registry (step 1a) where he adds information on his repository, such as title, description, contact person, the supported protocols used in his repository (OAI-PMH and DC metadata), etc. The registry uses an RSS feed to alert all (step 1.2) of its client tools about the new repository that has been added. In response to this alert, the ARIADNE harvester automatically issues a query (step 2) to the registry to get all relevant information about this repository. In step 3, the harvester performs the following steps:

- The DC metadata is harvested from provider X using the OAI-PMH protocol (step 3.1).

- From experience in different projects, we have learned that we cannot rely on the identifiers that are added by content providers because they are e.g. integers or descriptive titles. In a vast network of repositories, these are not 


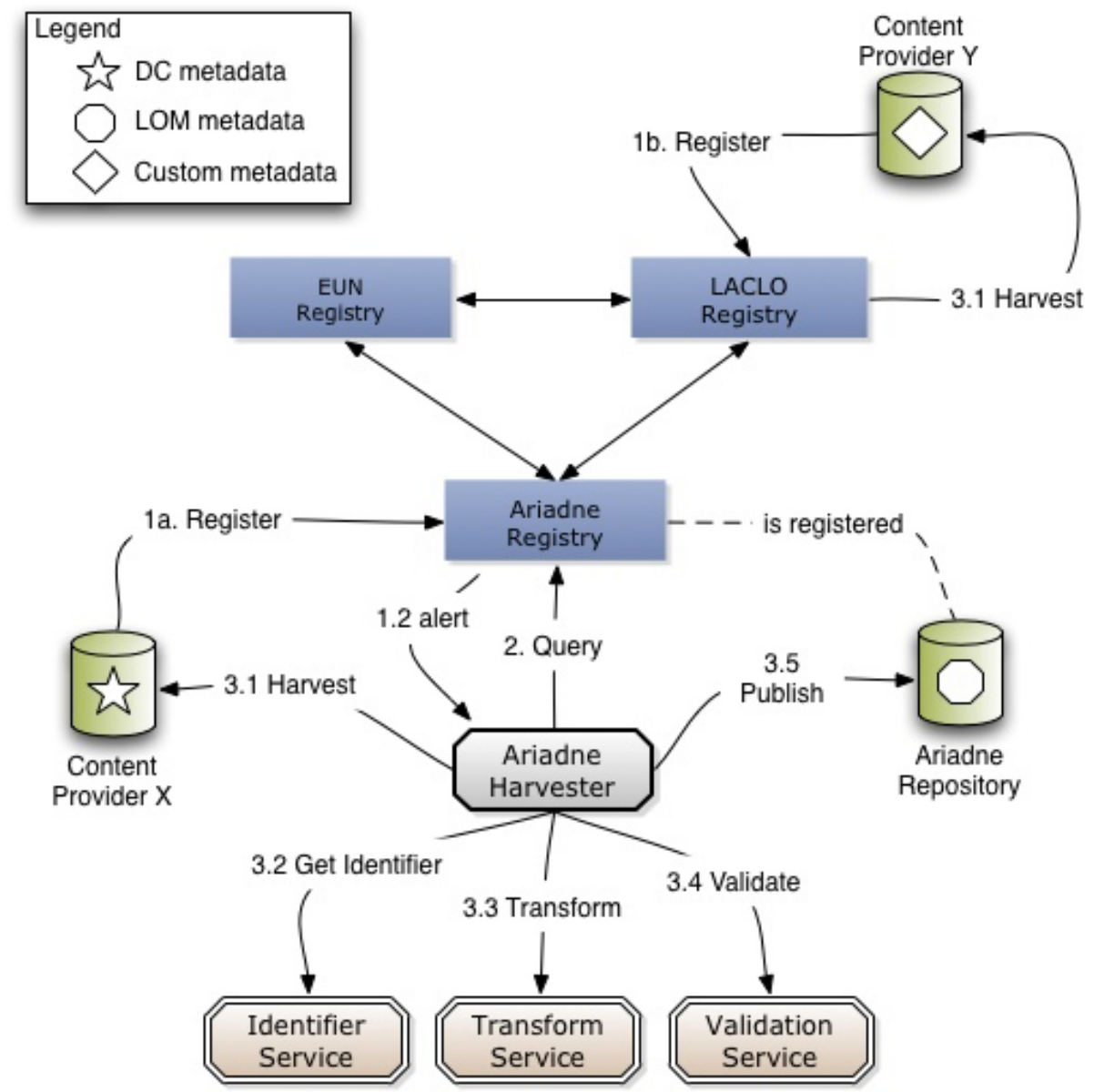

Fig. 2. From Content Provider to GLOBE

good identifiers because duplicates may arise. That's why the harvester calls the Identifier service (step 3.2) to generate a unique identifier and it adds those on-the-fly to the metadata instances of provider $\mathrm{X}$.

- A call to the transform service takes care of the conversion from DC to IEEE LOM, the standard used in both the ARIADNE and the GLOBE network to describe learning resources. (step 3.3).

- At the moment, the GLOBE network brings together content from over 70 repositories. Mapping metadata from a local metadata scheme to a global one typically happens happens either by manually editing one record at the time, or in batch with a script written specifically for this mapping. Our experience has shown that both of these methods make this a very error-prone process. For example, in the eContentplus project MELT 22, there were originally over $90 \%$ validation errors in content providers' metadata. The reports of the 
validation service helped the content providers to dramatically decrease this number to less than 5\%. Faulty metadata instances often render errors or inconsistencies in tools and UIs when searching for resources. All metadata instances are therefore validated against the validation scheme in use (step 3.4), and invalid instances are discarded.

- The harvester uses the SPI specification to publish the harvested and validated metadata into the ARIADNE repository which is registered as well in the registry and therefore interconnected with GLOBE. All content from provider X can thus be found in GLOBE (step 3.5) after the harvester has completed the cycle from steps 3.1 to 3.5. The content is available through an SQI target that is connected with the federated search layer of GLOBE, as well as through an OAI-PMH target that exposes the metadata again.

\section{Evaluation}

Software Quality Attributes are benchmarks that describe the intended behavior of a system within the environment for which it was built [9]. The ARIADNE infrastructure has been evaluated by common quality attributes such as performance, reliability, interoperability, configurability and scalability. In the following paragraph, we describe which quality attributes are considered important for ARIADNE and which tactics we follow for optimising these attributes.

- Interoperability is defined as the ability of two or more systems to cooperate at runtime. ARIADNE is a modular service-oriented architecture built on the principle that interoperability and extensibility is best achieved by the integration of different interfaces as clearly defined modules. These interfaces interoperate by using open and interoperable standards and specifications such as IEEE LOM, SQI, SPI and OAI-PMH. For example, SQI serves as a gateway to existing search protocols such as SRU/SRW, O.K.I OSIDs, etc. Another interoperability example is the transformation service that allows mapping metadata instances from one standard into another. A third example is the ARIADNE registry that currently contains around 50 repositories with different access protocols and metadata schemas.

- Scalability refers to the ability to support large numbers of components, or interactions among components, within an active configuration. Scalability in ARIADNE is achieved by distributing services across many components with each of them their specific focus such as the identifier, transform or validation service. Besides that, the ARIADNE validator ensures that our approach is scalable by avoiding the publishing of erroneous metadata into the repository. At the moment, ARIADNE exposes 822.126 valid metadata instances from both ARIADNE and GLOBE.

- Reliability relates to the amount of time for the system being up and running correctly. To ensure the reliability of our infrastructure, we have integrated CruiseControl, an extensible framework for creating a custom continuous build process [4, to monitor the uptime and responsiveness of all services. 
As a result, we are able to minimize the amount of time between failures. Furthermore, our tools and services are connected with a bug tracking system that allows users of e.g the ARIADNE finder to notify the technical support team when something is failing.

We have measured the degree to which the services are susceptible to failure by considering the presence of partial failures. The following calculations are based on the amount of time that the services were up and running correctly. By using the CruiseControl log data, we obtained the data required to analyze the reliability of ARIADNE services. This data has shown that all services have been up and running in $95 \%$ of the time during a period of 1 year, which is an acceptable outcome for service uptime. The log data also shows that most of the failures happened during the night which is due to the fact that during the day, technical partners are continuously aware of the system status.

- Performance measures the response time of the system to specific events. In the case of harvesting learning resources from content providers, the performance heavily depends on the performance of the OAI-PMH targets itself. For example, some providers might have been running the target on a slow machine which degrades the performance of a harvesting cycle. Besides that, performance is important for our tools on top of our repository such as the ARIADNE finder where we want to quickly find results in the repository if a users issues a query. To enable efficient, fast and facetted search, we make use of the combination of the open source Apache Lucene and SOLR frameworks. The authors from 23. show that Lucene performs well on keeping both the index size and the RAM usage small, and on providing powerful, accurate and efficient search algorithms.

Apache JMeter, a load testing tool [3], has been used to collect quantitative data about the performance of ARIADNE services. We have measured the response time of our search services on top of the GLOBE repository. Our test simulated 15 parallel users doing 100 queries each. Figure 3 shows the results of our test: the average response time of the services is around 300 milliseconds, with some busy peaks of less than 2 seconds. Those results are acceptable for now but we know from Shneiderman [29, that systems should achieve a rapid $100 \mathrm{~ms}$ interaction loop if we want the user perceived performance to be real good. Therefore, ARIADNE is currently investigating an in-memory search approach for further optimizing performance. First tests show us that this will allow ARIADNE to achieve a response time around $100 \mathrm{~ms}$ in the near future.

- Configurability refers to post-deployment modification of components, or configurations of components, such that they are capable of using a new service. We have shown in section 4 that it is easy to configure the combination of ARIADNE services considering the scenario that is needed. For example, when the need arises to convert a custom metadata format from a content provider into e.g. the ASPECT Application Profile of IEEE LOM; a new module can easily be plugged into the transformation service. Another example is that the harvester can easily be configured to not use the 


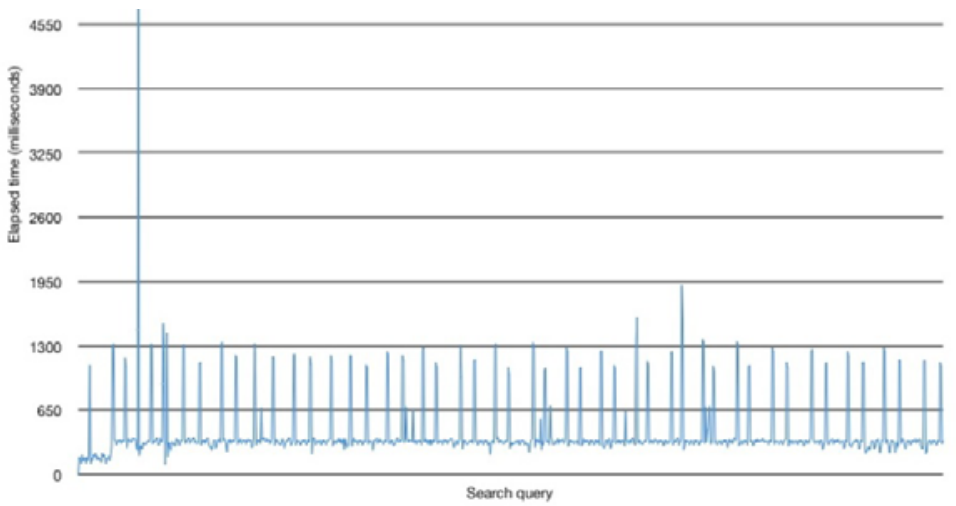

Fig. 3. Response time of ARIADNE Search service

transformation service if a content providers metadata is IEEE LOM. A third and last example is the storage layer; it is decoupled from the middle layer services which enables us to choose any database just by some small changes in a configuration file.

\section{Related Work}

As an open source, standards-based architecture for managing learning objects, ARIADNE has quite some technical commonalities with other architectures such as Fedora, LionShare, Edutella, and others. An extensive overview has been presented in [33. Besides the technical aspects, ARIADNE shares quite some commonalities with numerous organizations all over the world like LORNET in Canada [7, the Learning Resource Exchange (LRE) of European Schoolnet [5], MERLOT in the USA 20, The Open University of Japan 8, KERIS in Korea, III in Taiwan, LACLO in Latin America 6], etc. This list is far from exhaustive.

All of those use various technologies and metadata schemes but they all strive to be interoperable with each other to make shared online learning resources available to educators and students around the world. ARIADNE is one of the founding members of GLOBE, a global alliance that has committed to work collaboratively on this shared vision of ubiquitous access to quality educational content.

\section{Conclusion}

ARIADNE has been improving its services constantly. However, with the inclusion of a number of new services such as the registry service and the identification service, ARIADNE has now reached a level of maturity leading to a much greater uptake. 
The ARIADNE infrastructure has proven to be capable to provide flexible, effective and efficient access to large-scale collections. At the moment, ARIADNE exposes around a million learning resources through various repositories and networks of repositories that are registered in the ARIADNE registry. This number will undoubtedly increase in the coming months and years as more and more repositories are connecting to GLOBE.

This collection of learning resources allows for interesting research questions related to end users. Currently, we are focussing on accessing the learning material in various ways through information visualization [16, mobile information devices [26], multi-touch displays and mash-up applications.

In this paper, we have introduced a set of services which allows the integration and management of learning objects, described in various metadata schemas in numerous repositories across the globe. These services provide the backbone for an open learning infrastructure that provides access to impressive numbers of learning resources. As such, the abundance that we help to unlock will act as a platform for innovation by tool developers, trainers and teachers and learners themselves.

\section{Acknowledgements}

The work presented in this paper is partially supported by the European Commission eContentplus programme - projects ASPECT (ECP-2007-EDU-417008) and ICOPER (ECP-2007-EDU-417007).

\section{References}

1. Package exchange notification services (pens), http://www. aicc.org/docs/AGRs/agr011v1.pdf

2. Simple web-service offering repository deposit (sword), http://swordapp.org/

3. Apache jmeter (April 2010), http://jakarta.apache.org/jmeter

4. Cruisecontrol (April 2010), http://cruisecontrol.sourceforge.net/

5. European schoolnet, http://www.eun.org/ (last retrieved: April 2010)

6. The latin american community of learning objects (laclo), http://www.laclo.org/ (last retrieved: April 2010)

7. Lornet, www.lornet.ca (last retrieved: April 2010)

8. Open university of japan, http://www.u-air.ac.jp/eng/index.html (last retrieved: April 2010)

9. Bass, L., Clements, P., Kazman, R.: Software Architecture in Practice, 2nd edn. SEI Series in software engineering. Addison-Wesley Professional, Reading (June 2006) ISBN 0321154959

10. Baumgartner, P., Vuorikari, R., Van Assche, F., Duval, E., Zens, B., Massart, D., Vandeputte, B., Mesdom, F.: Experiences with the learning resource exchange for schools in europe. elearningeuropa.info (December 2009)

11. Duval, E., Hodgins, W.: Standardized uniqueness: Oxymoron or vision of the future? Computer 39(3), 96-98 (2006) 
12. Massart, D., Smith, N., Tice, R.: D2.2 design of data model and architecture for a registry of learning object repositories and application profiles (2008), http://www . aspect-project.org/sites/default/files/docs/ASPECT_D2p2.pdf

13. Globe. The globe consortium (2008), http://globe-info.org/globe/go (last retrieved: April 2008)

14. IEEE. IEEE Standard for Learning Object Metadata. The Institute of Electrical and Electronics Engineers, Inc., 3 Park Avenue, New York, NY 10016-5997, USA (September 2002)

15. ISO/IEC. Iso/iec 11578:1996 information technology - open systems interconnection - remote procedure call (1996), http://www.iso.ch/cate/d2229.html

16. Klerkx, J., Verbert, K., Duval, E.: Visualizing reuse: more than meets the eye. In: Tochtermann, K., Maurer, H. (eds.) Proceedings of 7th International Conference on Knowledge Management, Graz, Austria, pp. 389-396 (2006)

17. Lee, D., Chu, W.W.: Comparative analysis of six xml schema languages. SIGMOD Rec. 29(3), 76-87 (2000)

18. Manouselis, N., Najjar, J., Kastrantas, K., Salokhe, G., Stracke, C., Duval, E.: Metadata interoperability in agricultural learning repositories: an analysis. Computers and Electronics in Agriculture 70(2), 302-320 (2010)

19. McCallum, S.: A look at new information retrieval protocols: Sru, opensearch/a9, cql, and xquery. Library of Congress, USA (2006)

20. McMartin, F.: Case study: MERLOT: A model of user involvement in digital library design and implementation. Journal of Digital Information 5(3) (September 2004)

21. Meire, M., Ochoa, X., Duval, E.: Samgi: Automatic metadata generation v2.0. In: AACE (ed.) Proceedings of World Conference on Educational Multimedia, Hypermedia and Telecommunications, pp. 1195-1204 (2007)

22. MELT. the melt project (2008), http://www.melt-project.eu

23. Middleton, C., Baeza-Yates, R.: Technical report: A comparison of open source search engines. Technical report, Universitat Pompeu Fabra, Barcelona, Spain (November 2008)

24. Najjar, J., Wolpers, M., Duval, E.: Contextualized attention metadata. D-Lib Magazine 13(9/10) (2007)

25. Ochoa, X., Duval, E.: Use of contextualized attention metadata for ranking and recommending learning objects. In: CAMA 2006: Proceedings of the 1st international workshop on Contextualized attention metadata: collecting, managing and exploiting of rich usage information, pp. 9-16. ACM, New York (2006)

26. Parra, G., Duval, E.: More! a social discovery tool for researchers. In: Proceedings of ED-MEDIA 2010-World Conference on Educational Multimedia, Hypermedia \& Telecommunications (2010) (accepted)

27. PILIN Project. Pilin project: Project closure report (December 2007), https://www.pilin.net.au/Closure_Report.pdf

28. Safehaus. Java uuid generator(jug) (April 2010), http://jug.safehaus.org/Home

29. Shneiderman, B., Bederson, B.B.: The Craft of Information Visualization: Readings and Reflections. Morgan Kaufmann Publishers Inc., San Francisco (2003)

30. Simon, B., Massart, D., van Assche, F., Ternier, S., Duval, E., Brantner, S., Olmedilla, D., Miklos, Z.: A Simple Query Interface for Interoperable Learning Repositories. In: Proceedings of the 1st Workshop on Interoperability of Web-based Educational Systems, pp. 11-18 (2005)

31. Van De Sompel, H., Nelson, M.L., Lagoze, C., Warner, S.: Resource harvesting within the oai-pmh framework. D-Lib Magazine 10(nb. 12) (December 2004) 
32. Demidova, E., Olmedilla, D., Memmel, M., Ternier, S., Massart, D., Duval, E.: D4.8 spi - the simple publishing interface. Technical report, Prolearn

33. Ternier, S., Verbert, K., Parra, G., Vandeputte, B., Klerkx, K., Duval, E., Ordonez, V., Ochoa, X.: The ariadne infrastructure for managing and storing metadata. IEEE Internet Computing 13(4), 18-25 (2009)

34. Ternier, S., Massart, D., Campi, A., Guinea, S., Ceri, S., Duval, E.: Interoperability for searching learning object repositories, the prolearn query language. D-Lib Magazine 14(1/2) (January/February 2008)

35. Verbert, K., Duval, E.: Alocom: a generic content model for learning objects. International Journal on Digital Libraries 9(1), 41-63 (2008)

36. CEN WS-LT. The simple publishing interface specification (cwa 16097), ftp://ftp.cen.eu/CEN/Sectors/TCandWorkshops/Workshops/CWA16097.pdf 